\title{
MODELING THE SEISMICITY OF CENTRAL IONIAN ISLANDS WITH SEMI-MARKOV MODELS
}

\section{Pertsinidou C.E. ${ }^{1,2}$, Tsaklidis G. ${ }^{1}$, Limnios N. ${ }^{2}$ and Papadimitriou E. ${ }^{3}$}

${ }^{1}$ Aristotle University of Thessaloniki, Department of Mathematics, 54124, Thessaloniki, Greece, tsaklidi@math.auth.gr

${ }^{2}$ LMAC Laboratoire de Mathématiques Appliquées de Compiègne EA 2222, Sorbonne universités,

Université de Technologie de Compiègne - CS 60319 - 60203 Compiègne Cedex, France, p.eli.christina@gmail.com,nikolaos.limnios@utc.fr

${ }^{3}$ Aristotle University of Thessaloniki, Geophysics Department, 54124, Thessaloniki, Greece, ritsa@geo.auth.gr

\begin{abstract}
Earthquakes with $M \geq 5.2$ that occurred in the area of central Ionian Islands (19112014) are assumed to form a semi-Markov chain, aiming to contribute in the field of seismic hazard assessment. The sojourn times are considered to be geometric or approximated by Pareto distributions. Destination probabilities are examined and the results demonstrate that in many cases these probabilities become higher adequately forecasting the magnitude class of an anticipated earthquake. The geometrically distributed model can also reveal the more probable occurrence time of the next earthquake since for this model the destination probabilities were found to obtain many times their maximum values for the real occurrence time. The successful forecasting as for the occurrence time is $63.75 \%$ for all earthquakes and becomes $71.42 \%$ for the larger magnitude events $(M \geq 6.0)$.
\end{abstract}

Keywords: Interevent times, destination probabilities, geometric distribution, Pareto distribution.

\section{Пєрíi $\eta \psi \eta$}

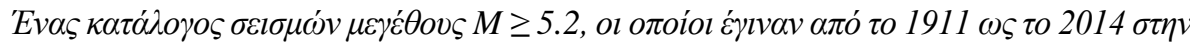

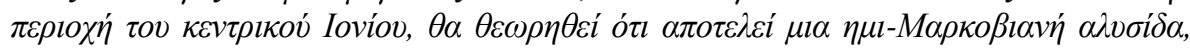

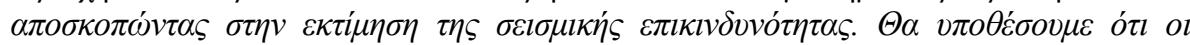

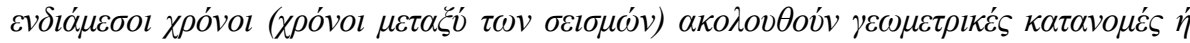

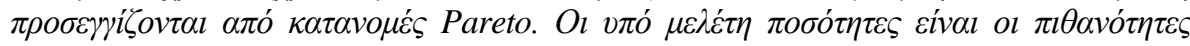

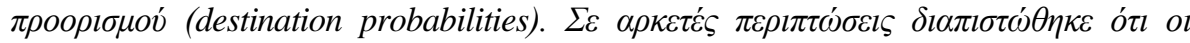

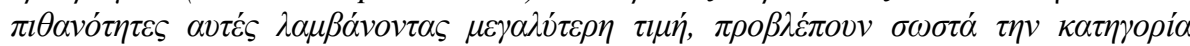

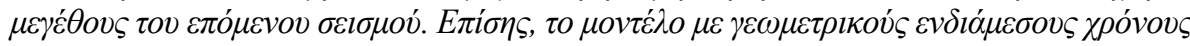

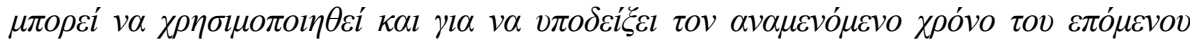

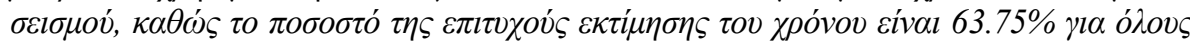

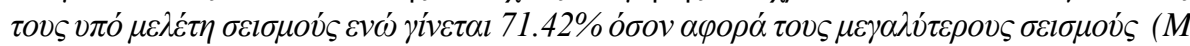
$\geq 6.0$ ).

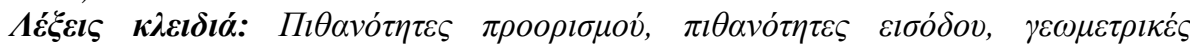

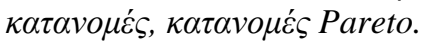




\section{Introduction}

A semi-Markov model can describe the evolution of a system in time where the current state of the system depends only upon the previous one and the sojourn times can be arbitrarily distributed (Barbu and Limnios, 2008). These models have been applied in many fields such as finance, meteorology, neuroscience and biology. Research has been conducted also in Seismology aiming to forecast an anticipated earthquake. For example, a semi-Markov model was applied (Altinok and Kolcak, 1999) in the north Anatolian fault zone between the longitudes $26.00^{\circ} \mathrm{E}$ and $42.00^{\circ} \mathrm{E}$ using a dataset of seventy one earthquakes with $\mathrm{M} \geq 5.5$ that occurred in the area between 1902 and 1992. The evaluated magnitude to magnitude interval transition probabilities demonstrate that the probability that an earthquake with $5.5 \leq \mathrm{M}<6.0$ will follow another one of the same magnitude range, decreases as the time increases, whereas the probability that an earthquake with $5.5 \leq \mathrm{M}<6.0$ will follow another one of $6.0 \leq \mathrm{M}<6.5$ or $6.5 \leq \mathrm{M}$ increases with time. The study area was also divided in three subareas and the estimated region to region transition probabilities were found to increase with time.

Semi-Markov modelling was adopted to describe 223 datasets each one containing one mainshock and the corresponding sequence of aftershocks. The data were extracted randomly from the seismic database "Commissariat à l'Energie Atomique" from different regions around the world covering the period 1984 to 1989 (Al-Hajjar and Blanpain, 1997). The main event and the aftershocks were classified into four classes $5.5 \leq \mathrm{M} 1<6.5,4.5 \leq \mathrm{M} 2<5.5,3.5 \leq \mathrm{M} 3<4.5$ and $3.5<4$. The scope of the study was to estimate the time duration $T$ of the aftershock activity. For mainshocks of category $M 1$ the evaluated time duration was $\mathrm{T}=130$ days and for mainshocks of class M2 the time duration was found to be $\mathrm{T}=58$ days.

A discrete semi-Markov model was applied on major seismic zones of the circum-Pacific belt for examining the recurrence of large earthquakes $M \geq 7.8$ (Patwardha et al., 1980). Historical seismicity data were taken into consideration and the results presented reasonable agreement between the calculated and actual waiting times as well as the calculated and actual earthquake magnitudes. Semi-Markov models were also developed (Sadeghian et al., 2008) to forecast the three parameters (time, location and magnitude) of the next earthquake occurrences with $M \geq 6.0$ in Iran. The study area was divided into zones (states of the model) and after declustering 3179 earthquakes between 1973 and 2007 were used and classified into 5 categories with magnitudes ranging from M $\leq 3.6$ to $6.3<\mathrm{M}$. The corresponding probability matrices were evaluated and $42 \%$ of the last 179 earthquakes belonging in the magnitude classes $\mathrm{M} 1 \leq 3.6,3.6<\mathrm{M} 2 \leq 4.8$ and $4.8<\mathrm{M} 3 \leq 5.4$ were correctly forecasted.

The forecasting ability of the semi-Markov model was also tested (Sadeghian, 2012) in the Tehran province area using as data earthquakes of all magnitudes classified into three categories $M<4.5,4.5 \leq M \leq 5$ and $5<M$ from 1966 to 2004. Considering transitions from region-to-region (the study area was divided into 6 regions) and magnitude-to-magnitude the obtained results demonstrate that 56\% of the earthquakes that occurred from 2004 and on have been correctly forecasted in all the three dimensions, $19 \%$ have been forecasted only as for their location and $19 \%$ have been forecasted as for the magnitude and location.

A continuous-time semi-Markov model has been applied to a dataset of earthquakes with $\mathrm{M} \geq 5.5$ that occurred from 1953 to 2007 in the region of the Northern Aegean Sea, which accommodates high seismic activity (Votsi et al., 2012). Earthquake occurrence rates were evaluated and it was found that the probability of an earthquake occurrence for a given state $j$ (the states refer to the magnitude) increases as time elapses. A new classification of states combining both magnitude and fault orientation was also proposed, dividing the study area into smaller subareas on the basis of faulting similarity. The hitting time of an earthquake occurrence with $\mathrm{M} \geq 5.6$ into each one of the subareas was calculated and indicated that all the subregions presented a similar behaviour with regard to the particular estimated quantity. 


\section{Data and area under study}

The study area includes Kefalonia and Lefkada islands, and is characterized by frequent occurrence of strong $(M \geq 6.0)$ earthquakes. The Kefalonia transform fault system runs along the western coasts of these Islands and connects continental collision to the north with subduction to the south. A belt of thrust faulting, with a NE-SW direction of the axis of maximum compression, runs along the eastern coastline of Adriatic Sea, and terminates just north of the Lefkada Island. The subduction of the eastern Mediterranean oceanic floor beneath the Aegean microplate, which starts south of Kefalonia Island, forms the Hellenic Arc (Papazachos and Comninakis, 1971), which is the most prominent tectonic feature of the Aegean region. The dextral strike-slip character of the Kefalonia transform fault was first evidenced by Scordilis et al. (1985) and it is recognized as a major discontinuity between the Apulian platform and the West Hellenic arc with special emphasis to its prolongation towards the Ionian Abyssal Plain (Kokinou et al., 2006). The central Ionian Islands area constitutes the most active zone of shallow seismicity in the broader Aegean region. It is a key area for long-term study of the earthquake process since it has both a high level of seismic activity along an active boundary and a record of earthquakes extending back at least to 1850 for events at M 6 minimum magnitude of completeness (Papadimitriou, 2002).

For this research we use a complete catalogue of earthquakes from 1911 to 2014 with $\mathrm{M} \geq 5.2$. A semi-Markov model, with time unit of one month, will be applied. In the case when more than one earthquake exists in the same time unit, only the one with the higher magnitude is considered. The total number of events is 80 and the data are classified into two categories, i.e., state 1: $5.2 \leq \mathrm{M}<$ 6.0 and state $2: M \geq 6.0$.

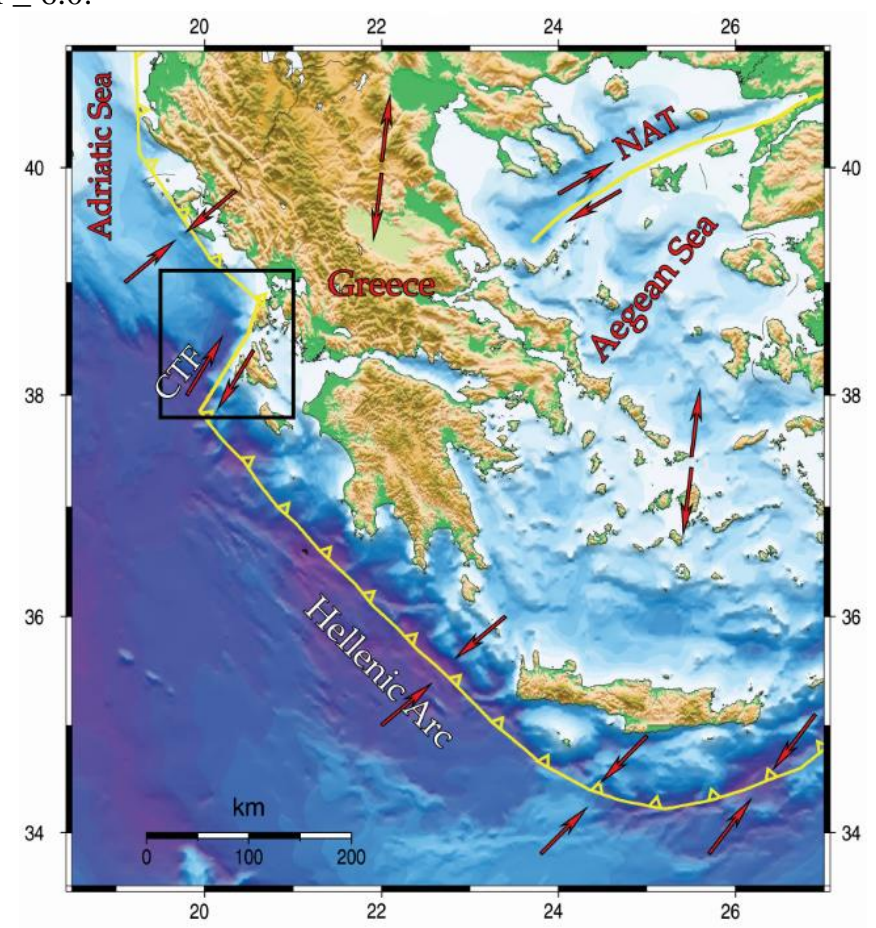

Figure 1 - The main geodynamic features of the broader Aegean region shown on a relief ma p. The active boundaries are shown as solid lines. The arrows indicate the approximate direc tion of relative plate motion. NAT: North Aegean Trough, CTF: Cephalonia Transform Faul t. The rectangle indicates the study area. 
The above classification allows more data to be included in each category, which is important from a statistical point of view. It is also based on how destructive an earthquake could be, since magnitudes larger than 6.0 can cause severe damage in the study area.

\section{Semi-Markov framework}

Let us consider a random system with finite state space $E=\{1, \ldots, d\}$. We suppose that the evolution of the system in time is described by the following chains (Barbu and Limnios, 2008):

The embedded Markov chain $J=\left(J_{n}\right)_{n \in N}$ with state space $E$, where $J_{n}$ is the system's state at the $n$th jump time ( $n$th earthquake occurrence).

The chain $S=\left(S_{n}\right)_{n \in N}$ with state space $\mathbb{N}$, where $S_{n}$ denotes the $n$th jump time. We suppose that $S_{0}=0$ and $0<S_{1}<S_{2}<\ldots<S_{n}<S_{n+1}<\ldots$

The chain $X=\left(X_{n}\right)_{n \in N}$, where $X_{n}:=S_{n}-S_{n-1}$ for all $n \in \mathbb{N}^{*}$ and $X_{0}:=0$. Thus, $X_{n}$ represents the sojourn time in state $J_{n-1}$ before the $n$th jump (it is the time between successive earthquakes). We also denote by $P=\left(p_{i j}\right)_{i, j \in E}$ the transition matrix of $J$, defined by $p_{i j}:=\mathbb{P}\left(J_{n+1}=j \mid J_{n}=i\right), i, j \in E, n \in \mathbb{N}$.

and let $f_{i j}(\cdot)$ be the common probability function of $X_{n+1}, n \in \mathbb{N}$, conditioned on $J_{n}$ and $J_{n+1}$ : $f_{i j}(k):=\mathbb{P}\left(X_{n+1}=k \mid J_{n}=i, J_{n+1}=j\right), k \in \mathbb{N}$.

The chain $Z=\left(Z_{k}\right)_{k \in N}$ is considered to be a semi-Markov chain associated with the couple $(J, S)$ if $Z_{k}:=J_{N(k)}, k \in \mathbb{N}$, where $N(k):=\max \left\{n \in \mathbb{N} \mid S_{n} \leq k\right\}$ is the discrete-time counting process of the number of jumps in $[1, k]$.

Starting from a semi-Markov sample path we define for any states $i, j$ and any positive integer $k$, $1<k \leq M$ :

- $\quad N_{i}(M)$ : the number of visits to state $i$ of the Embedded Markov Chain (EMC), up to time $M$;

- $\quad N_{i j}(M)$ : the number of transitions of the EMC from $i$ to $j$, up to time $M$;

The "approached maximum-likelihood estimator" of the $(i, j)$ element of the transition matrix $\mathrm{P}$ is $\hat{p}_{i j}(M)=N_{i j}(M) / N_{i}(M)$. Since $f_{i j}(k)$ is the sojourn time distribution function, the complement ary cumulative sojourn time distribution is defined as (Howard, 1971)

${ }^{>} f_{i j}(k)=\sum_{m=k+1}^{\infty} f_{i j}(m), k=0,1,2, \ldots$.

Now let $e_{i j}(z \mid u)$ be the probability that a process which enters state $i$ at time zero and at transitio $\mathrm{n}$ zero will enter state $j$ at time $u$ on its $z$-th transition. It is (Howard, 1971), $e_{i j}(z \mid u)=P\left\{\mathrm{U}(u)=z, \mathrm{U}(u-1)=z-1, J_{n}=j \mid J_{0}=i, t(0)=0\right\}, i, j,=1,2, \ldots, N, z, u=0,1,2, \ldots$ where $\mathrm{U}(u)$ denotes the number of transitions until time $u$ and $t(0)$ the starting time.

This probability is called entrance probability and it is given by the following recursion

$e_{i j}(z \mid u)=\delta_{i j} \delta(u) \delta(z)+\sum_{r=1}^{N} \sum_{m=1}^{u} p_{i r} f_{i r}(m) e_{r j}(z-1 \mid u-m)$

where

$\delta_{i j}=\left\{\begin{array}{l}1 \text { if } i=j \\ 0 \text { if } i \neq j\end{array}\right.$ and $\delta(z)=\left\{\begin{array}{l}1 \text { if } z=0 \\ 0 \text { elsewhere }\end{array}\right.$ 
and $e_{i j}(0 \mid u)=\delta_{i j} \delta(u)$. The quantity of interest in this paper is the destination probability denoted by $\gamma_{i j q}(z \mid u)$ and defined to be the probability that a process which was in state $i$ at time 0 at transition 0 is observed to be in state $j$ at time $u$ after $z$ transitions by that time, and will make its next transition to state $q$. The following recursion demonstrates that the destination probability $\gamma_{i j q}(\cdot \mid \cdot)$ is the convo lution of the entrance probability $e_{i j}(\cdot \mid \cdot)$ and the complementary cumulative sojourn time distributio $\mathrm{n}^{>} f_{j q}(u-m)$ multiplied by the transition probability $p_{j q}$ (Howard, 1971) that is $\gamma_{i j q}(z \mid u)=\sum_{m=1}^{u} e_{i j}(z \mid m) p_{j q}>f_{j q}(u-m), i, j, q=1,2, \ldots N, z, u=1,2, \ldots$

\section{Semi-Markov model with geometric sojourn times}

It will be now assumed that the sojourn times of the model follow geometric distributions of the form

$f_{i j}(k)=\left(1-a_{i j}\right)^{k-1} a_{i j}, \quad k \in\{1,2,3, \ldots\}$ where $0<a_{i j}<1$ is the success probability.

The transition probability matrix estimated by our data, described in section 2 , is

$\boldsymbol{P}=\left(\begin{array}{ll}0.7705 & 0.2295 \\ 0.6842 & 0.3158\end{array}\right)$.

The sojourn time values evaluated from the data can be observed in Table 1.

The maximum likelihood estimation for the parameter of the geometric distribution is $\hat{a}_{i j}=1 / \bar{X}_{n}$

where $\bar{X}_{n}$ represents the mean value of the sojourn times. Hence,

$f_{11}(k)=(1-0.0561)^{k-1} 0.0561, \quad f_{12}(k)=(1-0.0617)^{k-1} 0.0617$,

$f_{21}(k)=(1-0.1057)^{k-1} 0.1057, f_{22}(k)=(1-0.1724)^{k-1} 0.1724$.

The matrix of the complementary cumulative geometric sojourn time distributions is

$$
\begin{aligned}
& >F(k)=\left(\begin{array}{ll}
(1-0.0561)^{k} & (1-0.0617)^{k} \\
(1-0.1057)^{k} & (1-0.1724)^{k}
\end{array}\right) \text {, where the } i, j \text { elements are given by the equation } \\
& { }^{>} f_{i j}(k)=\sum_{m=k+1}^{\infty}\left(1-a_{i j}\right)^{(m-1)} a_{i j}=\left(1-a_{i j}\right)^{k} .
\end{aligned}
$$

Table 1 - Sojourn times evaluated from the data (in months).

\begin{tabular}{|l|l|}
\hline $\begin{array}{l}\text { Transition from state } \\
\boldsymbol{i} \text { to state } \boldsymbol{j}(\boldsymbol{i}, \boldsymbol{j})\end{array}$ & \multicolumn{1}{|c|}{ Sojourn times $\boldsymbol{X}_{\boldsymbol{n}}$} \\
\hline \multirow{3}{*}{$(1,1)$} & $1,5,24,42,5,19,39,10,57,12,60,26,3,2,13,22,22,8,3,1$, \\
& $\begin{array}{l}3,43,2,9,8,5,37,13,13,13,51,13,1,27,18,15,4,11,29,25, \\
10,14,51,31,6,3,9\end{array}$ \\
\hline$(1,2)$ & $8,2,3,18,17,17,1,25,9,17,19,1,8,82$ \\
\hline$(2,1)$ & $2,4,6,4,12,1,1,1,63,1,1,2,25$ \\
\hline$(2,2)$ & $2,21,2,3,1$ \\
\hline
\end{tabular}

Before proceeding in the evaluations we will test whether the sojourn times (Table 1) follow the aforementioned geometric distributions. For this purpose the Mann-Whitney (Mann and Whitney, 
1947) and Anderson-Darling (Anderson and Darling, 1952) goodness of fit tests will be implemented (Dodge, 2008). The null hypothesis is rejected ${ }^{(} H_{0}:$ the data come from a certain distribution), at the significance level $\alpha$ if the evaluated $p$-value is smaller than $\alpha$ (for this application $\alpha=0.05$ ). Applying the Mann-Whitney test via the SPSS package and the Anderson -Darling test via the R package "ADGofTest" (Bellosta, 2011) we derive the following results.

Table 2 - Testing whether the sojourn times come from the geometric distribution.

\begin{tabular}{|c|c|c|c|c|}
\hline Sojourn times & \multicolumn{4}{|c|}{$\boldsymbol{X}_{\boldsymbol{n}}$} \\
\hline states & $(1,1)$ & $(1,2)$ & $(2,1)$ & $(2,2)$ \\
\hline Mann-Whitney $U$ & 2.5 & 0 & 2.000 & 1.000 \\
\hline$p$-value & 0.767 & 0.221 & 0.245 & 1.000 \\
\hline Anderson-Darling AD & 0.9595 & 0.5329 & 1.5123 & 0.8147 \\
\hline$p$-value & 0.3788 & 0.7105 & 0.1738 & 0.4634 \\
\hline
\end{tabular}

Observing Table 2 we realize that for all the cases the $p$-values are found to be greater than $\alpha=0.05$. Hence, there is not much evidence to reject the null hypothesis.

It will be now considered that we start to observe the process on 24-05-1911 (M=5.3, $i=1)$. We know that the next earthquake occurred on 24-01-1912 $(\mathrm{M}=6.8, j=2)$ and the time elapsed between these events is $u=8$ while there was only one transition $(z=1)$. We seek the probability that the next earthquake will be of state $q=1$ or $q=2$. The corresponding destination probabilities are $\gamma_{121}(1 \mid 8)=0.0422$ and $\gamma_{122}(1 \mid 8)=0.0154$. Therefore, the probability that the next earthquake will be of state 1 is 2.74 times higher than the probability of an earthquake occurrence of state 2 . Observing o ur data, we realize that the next earthquake was of state 1 which was the most probable to occur.

Let us consider that we start to observe the process again on 24-05-1911. The second transition $(z=2)$ is on 05-03-1912 when $j=1$ and the elapsed time is $u=10$ months. We seek the probability tha $t$ the next earthquake will be of state 1 or state 2 . The corresponding quantities are $\gamma_{111}(2 \mid 10)=0.06$ $\gamma_{112}(2 \mid 10)=0.0175$ which means that the probability that the next earthquake will be of state 1 is 3 .42 times higher than the probability that the next earthquake will be of state 2 . Observing the data we rea- lize that the next earthquake is of state 1 which was the most probable to occur.

In the same way $\gamma_{111}(4 \mid 16)=0.00978$ and $\gamma_{112}(4 \mid 16)=0.00287$ which means that if the starting ti me is on 24-05-1911 when $i=1$ and we observe the process on 28-09-1912 when $j=1$, the time elapsed between $i$ and $j$ is $\mathrm{u}=16$ months while the process has made 16 transitions, then the probability that th e next earthquake will be of state 1 is 3.4 times higher compared to the probability that the next earth quake will be of state 2 , which describes adequately the succession of the data.

As an example we will now present a different use of the destination probabilities. Let us assume $\mathrm{t}$ hat we start to observe the chain on 10-04-1962 when an earthquake of $M=6.3$ occurred ( $i=2)$. We would like to evaluate the destination probabilities for the next two earthquakes considering that $\mathrm{w}$ e do not know neither their magnitude nor their occurrence time.

The above figure 2 demonstrates e.g. for $\mathrm{u}=3$, that in the next 3 months it is more probable that an earthquake of state 1 will occur (green color) and will be also followed by an earthquake of state 1 . For the next three months there exists also a higher probability (dark blue color) that an earthquak e of state 2 will occur and will be followed by one of state 1 (this correspond to the observed data). The destination probabilities $\gamma_{212}(1 \mid u)$ and $\gamma_{222}(1 \mid u)$ illustrated by orange and light blue color res pectively, are much smaller. 


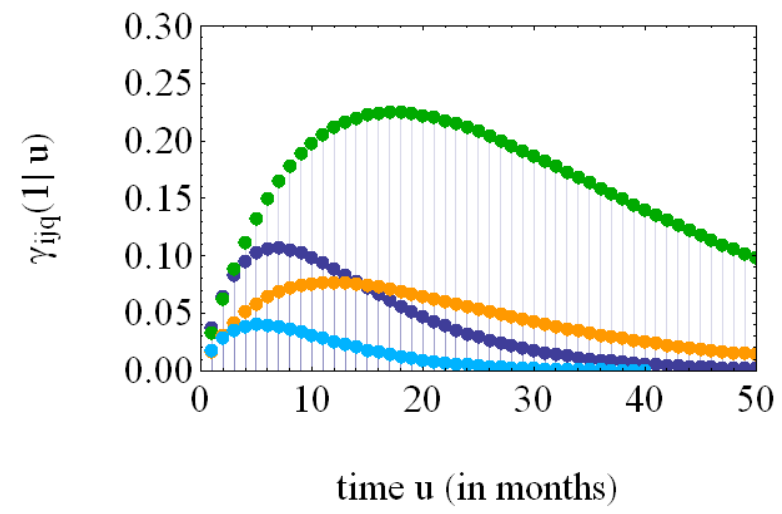

Figure 2 - Destination probabilities green: $\gamma_{211}(1 \mid u)$, dark blue: $\gamma_{221}(1 \mid u)$, orange: $\gamma_{212}(1 \mid u)$, light blue: $\gamma_{222}(1 \mid u)$.

Now let 19-07-1961 be the starting time. The event of 19-07-1961 (M=5.3, $i=1)$ was followed by the events of 10-04-1962 (M=6.3) and 06-07-1962 $(M=6.1)$. We will evaluate the probability $\gamma_{122}(1 \mid u)$ aiming to reveal in how many months after the 7-1961 an earthquake of state 2 is anticipated for the $\mathrm{f}$ irst time. Figure 3 illustrates the probability of an earthquake of state 1 to be followed by two successi ve earthquakes of state 2 . The probability is very small, however, if this is the case then the first earth quake of state 2 (10-04-1962) is more probable to occur after 9 months (the curve of the graph has a maximum in 9 months) starting from 07-1961, and this fact corresponds to the data.

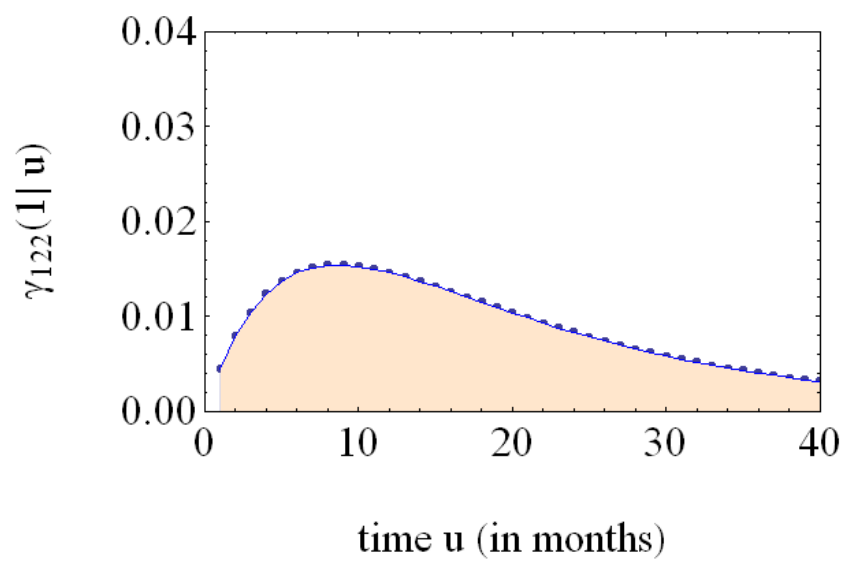

Figure 3 - Destination probabilities $\gamma_{122}(1 \mid u)$.

Since, the time of an earthquake occurrence is of great importance for the seismic hazard assessment, the above case will be tested for another event too. The earthquake of 09-12-2002 $(M=5.5, i=1)$ was $\mathrm{f}$ ollowed by the events of 14-08-2003 $(M=6.2, j=2)$ and 19-09-2005 (M=5.2, $q=1)$. Assuming that the starting point is on 09-12-2002 we will evaluate the probability $\gamma_{121}(1 \mid u)$ aiming to reveal in how $\mathrm{m}$ any months after the 12-2002, the event of state 2 is the most probable to occur.

According to the results illustrated in figure 4 the destination probability is very small. Nevertheless, $\mathrm{i}$ $\mathrm{f}$ this is the case then the event of state 2 is anticipated to occur more probably 10 months after the sta rting point. Indeed the time between the earthquakes of 09-12-2002 and 14-08-2003 is 8 months.

The aforementioned ability of the geometrically distributed model to forecast an earthquake occurrence time was tested for all the events of our dataset. More precisely, we assume that an event of state $i$ will be followed by another one of state $j$ after $u$ months (while the chain is considered to make its first 
transition, $z=1$ in $j$ ) and the earthquake of state $j$ will be followed by another one of state $q$. The aim is to reveal the time $u$ that the event of state $j$ is anticipated to occur.

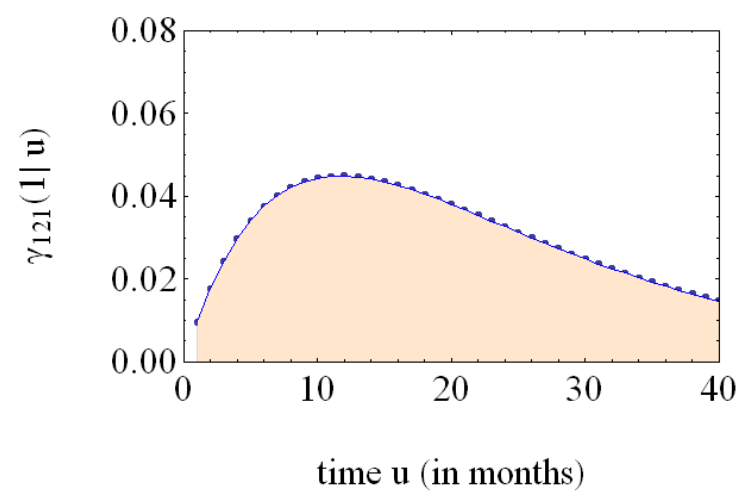

Figure 4 - Destination probabilities $\gamma_{121}(1 \mid u)$.

Table 3 - Estimation of the time $u$ (in months) that the earthquake of state $j$ will succeed the earthquake of state $i$.

\begin{tabular}{|l|c|c|c|c|c|}
\hline$\gamma_{\boldsymbol{i j q}}(\mathbf{1} \mid \boldsymbol{u})$ & $\boldsymbol{i}$ & $\boldsymbol{j}$ & $\boldsymbol{q}$ & $\boldsymbol{u}$ & $\boldsymbol{p}$ \\
\hline$\gamma_{121}(1 \mid u)$ & 1 & 2 & 1 & $3-25$ & $\mathrm{p}>0.03$ \\
\hline$\gamma_{211}(1 \mid u)$ & 2 & 1 & 1 & $4-25$ & $\mathrm{p}>0.18$ \\
\hline$\gamma_{111}(1 \mid u)$ & 1 & 1 & 1 & $4-40$ & $\mathrm{p}>0.14$ \\
\hline$\gamma_{112}(1 \mid u)$ & 1 & 1 & 2 & $5-37$ & $\mathrm{p}>0.04$ \\
\hline$\gamma_{122}(1 \mid u)$ & 1 & 2 & 2 & $3-20$ & $\mathrm{p}>0.01$ \\
\hline$\gamma_{221}(1 \mid u)$ & 2 & 2 & 1 & $2-17$ & $\mathrm{p}>0.06$ \\
\hline$\gamma_{212}(1 \mid u)$ & 2 & 1 & 2 & $4-25$ & $\mathrm{p}>0.05$ \\
\hline$\gamma_{222}(1 \mid u)$ & 2 & 2 & 2 & $2-10$ & $\mathrm{p}>0.03$ \\
\hline
\end{tabular}

For example the destination probabilities $\gamma_{121}(1 \mid u)$ in the second row of Table 3 demonstrate that there is a higher probability that the earthquake of state 1 will be followed by one of state 2 in the next 3-25 ( $>0.03)$ months under the assumption that the event of state 2 will be succeeded by another one of state 1 . The corresponding graphs are as follows.

The estimated time windows for the variable $u$, presented in Table 3 include 51 out of 80 real earthquake occurrence times (from our data) i.e., $63.75 \%$ of the total number of events. It is worth to be mentioned that when $j=2$ the aforementioned time windows contain 10 out of 14 earthquake occurrence times i.e., $71.42 \%$.

\section{Semi-Markov model with Pareto distributed sojourn times}

The Pareto distribution is a heavy tailed distribution, in the sense that the variables can take higher values with considerable probabilities, compared to other distribution functions. It is used to describe continuous data. In our case the sojourn times are considered to be discrete (time unit, one month). However, observing Table 1 it can be seen that the sojourn times of our application can take large values. This motivated us to use the Pareto distribution as an approximation. The probability density function (pdf) is $f(k)=\frac{a b^{a}}{k^{a+1}}, k \geq b, b>0, a>0$. 

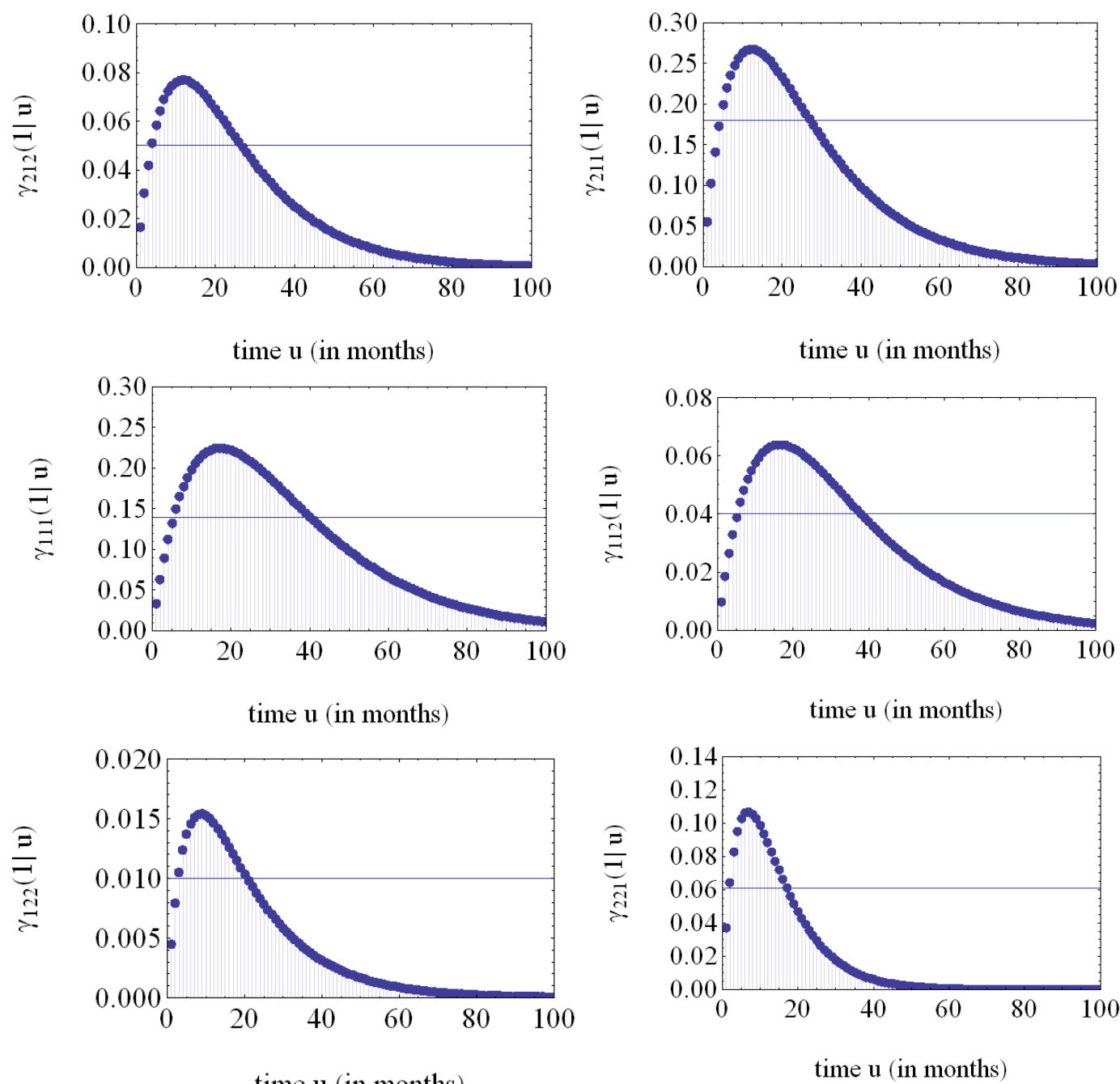

time $u$ (in months)

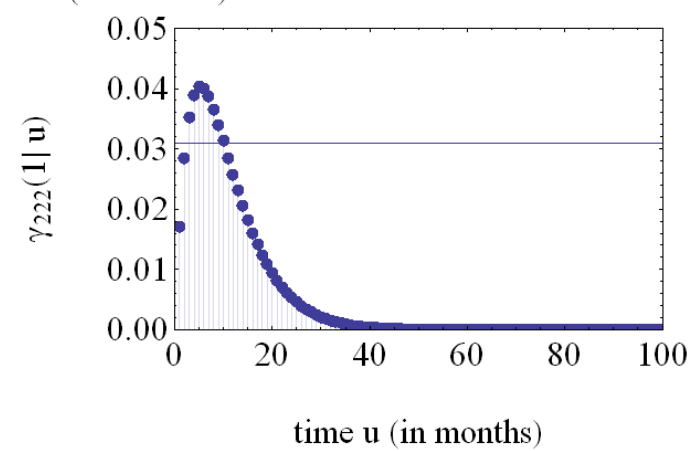

The maximum likelihood estimation of the parameters is (Malik, 1970)

$\hat{b}=\min _{i}\left(k_{i}\right), \quad(i=1,2, \ldots, n)$ and $\hat{a}=\frac{n}{\sum_{i}\left(\ln \left(k_{i}\right)-\ln \hat{b}\right)}$.

We obtain $\hat{a}_{11}=0.418, \hat{a}_{12}=0.465, \hat{a}_{21}=0.823, \hat{a}_{22}=0.904$ and for all $i, j, \hat{b}_{i j}=1$. Hence, 


$$
\begin{aligned}
& f_{11}(k)=\frac{0.418}{k^{0.418+1}}, \quad f_{12}(k)=\frac{0.465}{k^{0.465+1}}, \\
& f_{21}(k)=\frac{0.823}{k^{0.823+1}}, \quad f_{22}(k)=\frac{0.904}{k^{0.904+1}} .
\end{aligned}
$$

The complementary cumulative sojourn time distribution is of the form

$$
>^{\prime}(k)=\sum_{m=k+1}^{\infty} f(m)=\alpha b^{a} \zeta(1+a, 1+k)
$$

where $\zeta$ represents the Hurwitz Zeta function, defined as (Miller and Adamchik, 1998)

$$
\zeta(s, r)=\sum_{n=0}^{\infty} \frac{1}{(n+r)^{s}} \text {. }
$$

Thereby,

$$
{ }^{{ }} F(k)=\left(\begin{array}{ll}
0.418 \zeta(1.418,1+k) & 0.465 \zeta(1.465,1+k) \\
0.823 \zeta(1.823,1+k) & 0.904 \zeta(1.904,1+k)
\end{array}\right) .
$$

Before proceeding in the evaluation part we will test if the sojourn times of Table 1 can follow the estimated Pareto distributions. For this purpose the Mann-Whitney test will be implemented via the SPSS statistical package (The Anderson-Darling test cannot be applied for the Pareto distribution since for the estimated parameters $\hat{b}_{i j}$ the logarithm of the test cannot be defined).

Table 4 - Testing whether the sojourn times come from the Pareto distribution.

\begin{tabular}{|c|c|c|c|c|}
\hline Sojourn times & \multicolumn{4}{|c|}{$\boldsymbol{X}_{\boldsymbol{n}}$} \\
\hline states & $(1,1)$ & $(1,2)$ & $(2,1)$ & $(2,2)$ \\
\hline Mann-Whitney $U$ & 1 & 0 & 3 & 0 \\
\hline$p$-value & 0.248 & 0.221 & 0.439 & 0.221 \\
\hline
\end{tabular}

Observing Table 4 we realize that for all the cases the $p$-values are greater than $\alpha=0.05$, the signif i-cance level. Thus, there is not much evidence to reject the null hypothesis.

We consider now that the time we start to observe the process is on 24-05-1911 $(M=5.3, i=1)$ and it is known that the next earthquake occurred on 24-01-1912 $(M=6.8, j=2)$. The elapsed time is $u=8$ months and there was only one transition $(z=1)$. We seek the probability that the next earthquake will be of state $q=1$ or $q=2$. The corresponding evaluated quantities are $\gamma_{121}(1 \mid 8)=0.039$ and $\gamma_{122}(1 \mid 8)=0.0161$. This means that the probability that the next earthquake will be of state 1 is 2.41 times higher than the probability of an earthquake occurrence of state 2. Observing our data we realize that the next event was of state 1 which was the most probable to occur.

Let the starting time be again on 24-05-1911. We suppose now that we observe the process on 05 03-1912 when $j=1$ and the time between these events is $u=10$ months. The number of transitions that the process has made until state $j$ is $z=2$. We are interested in the probability that the next earthquake will be of state 1 or state 2 . The corresponding quantities are $\gamma_{111}(2 \mid 10)=0.247$ and $\gamma_{112}(2 \mid 10)=0.068$. Hence, the probability that the next earthquake will be of state 1 is 3.58 times higher than the probability of an earthquake occurrence of state 2. Observing our data, the next earthquake on 19-04-1912 is indeed of state $1(\mathrm{M}=5.5)$, which was the most probable to occur. 
In the same way $\gamma_{111}(4 \mid 16)=0.263$ and $\gamma_{112}(4 \mid 16)=0.0732$ which means that if the starting time is on 24-05-1911 when $i=1$ and we observe the process on 28-09-1912 when $j=1$, the time between $i$ and $j$ is $\mathrm{u}=16$ months and the process has made 16 transitions, then the probability that the next earthquake will be of state 1 is 3.59 times higher compared to the probability that the next earthquake will be of state 2 . Observing our data, we realize that indeed the next earthquake was of state 1 .

It will be now assumed that we start to observe the chain on 02-01-1940 while being in state $i=1$ $(M=5.2)$. We would like to evaluate the destination probabilities for the next two earthquakes, i.e., $\gamma_{112}(1 \mid u), \gamma_{111}(1 \mid u), \gamma_{121}(1 \mid u)$ and $\gamma_{122}(1 \mid u)$.

The probabilities $\gamma_{111}(1 \mid u)$ in Figure 5 are higher than all the other which means that it is more probable the next earthquake to be of state 1 at every time $u$ and also the following event to be again of state 1. This result corresponds to our data since the occurrences of 14-02-1943 and 22-05-1943 were both of state 1 . Figure 6 demonstrates the evaluation of the above probabilities under the assumption that the sojourn times follow geometric distributions.

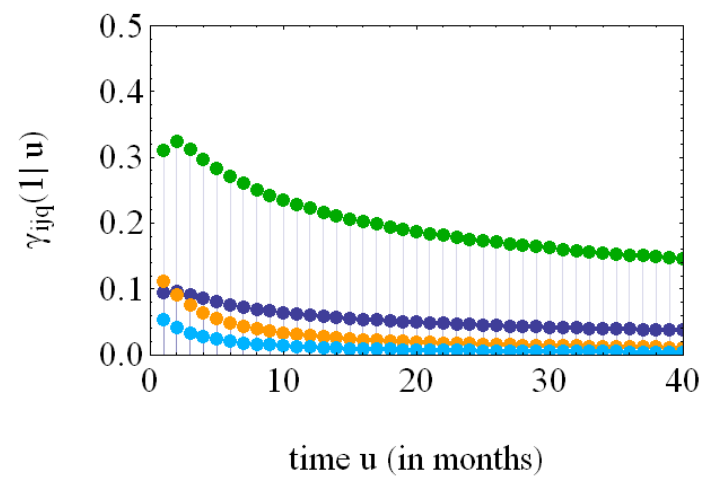

Figure 5 - Destination probabilities for the Pareto distributed sojourn times green: $\gamma_{111}(1 \mid u)$, dark blue: $\gamma_{112}(1 \mid u)$, orange: $\gamma_{121}(1 \mid u)$, light blue: $\gamma_{122}(1 \mid u)$.

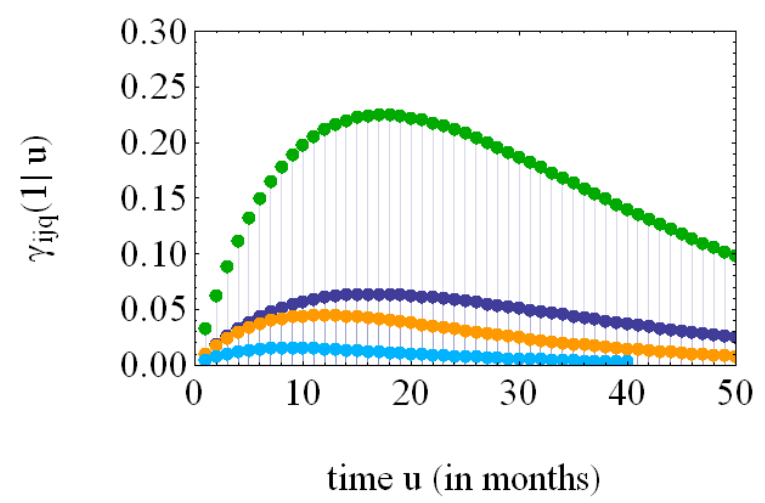

Figure 6 - Destination probabilities for the geometric sojourn times green: $\gamma_{111}(1 \mid u)$, dark blue: $\gamma_{112}(1 \mid u)$, orange: $\gamma_{121}(1 \mid u)$, light blue: $\gamma_{122}(1 \mid u)$.

The probabilities $\gamma_{111}(1 \mid u)$ are again higher compared to all the other. The advantage of the geometric distribution is that there is a maximum in the curve of the $\gamma_{111}(1 \mid u)$ showing that if the earthquake on 02-01-1940 is followed by another one of state 1 (14-02-1943) then this is more probable to occur in almost 20 months. Observing our data, we realize that the time $u$ is adequately approximated. The shape of the destination probability curves in figure 5 is not that helpful in the sense that they do not provide a maximum. In Figure 4 we find out that the curve of the destination 
probabilities $\gamma_{121}(1 \mid u)$ when the sojourn times are geometrically distributed, gives insight concerning the time in months that the earthquake of state 2 is anticipated, given that it will be followed by another one of state 1 . The corresponding probabilities for the Pareto distributed sojourn times are illustrated in Figure 7.

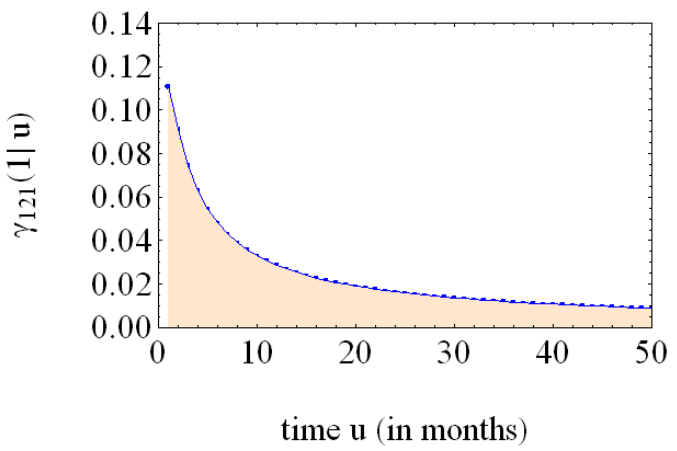

Figure 7 - Destination probabilities $\gamma_{121}(1 \mid u)$, Pareto distributed sojourn times.

Comparing Figures 4 and 7 we realize that the destination probabilities from the Pareto distribution are higher than these of the geometric but again they do not provide useful results as for the time (in months) that the next earthquake is more probable to occur.

\section{Conclusions}

A semi-Markov model with sojourn times that follow either geometric or Pareto distributions is applied to a dataset of earthquakes with $M \geq 5.2$ that occurred (1911-2014) in the central Ionian Islands. The evaluated destination probabilities were found to forecast adequately the magnitude class of an anticipated earthquake, taking higher values. The model with geometric distributed sojourn times gives insight to the real occurrence time of an earthquake since the estimated time windows with the higher probabilities include the real occurrence time for $63.75 \%$ out of all cases and provide $71.42 \%$ successful forecasting when the anticipated event is of $M \geq 6.0$.

\section{References}

Altinok, Y. and Kolcak, D., 1999. An application of the semi-Markov model for earthquake occurrences in North Anatolia, Turkey, Journal of the Balcan Geophysican Society, 2(4), 90-99.

Al-Hajjar, J. and Blanpain, O., 1997. Semi-Markovian approach for modelling seismic aftershocks, Engineering Structures, 19(12), 969-976.

Anderson, T.W. and Darling, D.A., 1952. Asymptotic Theory of Certain "Goodness of Fit" Criteria Based on Stochastic Processes, The Annals of Mathematical Statistics, 23(2), 193-212.

Barbu, V.S. and Limnios, N., 2008. Semi-Markov Chains and Hidden Semi-Markov Models toward Applications Their use in Reliability and DNA Analysis, Springer, 191.

Belosta, C.G., 2011. Anderson-Darling GoF test, R statistical package.

Dodge, Y., 2008. The Concise Encyclopedia of Statistics, Springer, 327-329.

Howard, R., 1971. Dynamic Probabilistic Systems, Volume II: Semi-Markov and Decision Processes, Jonh Wiley\& sons, New York.

Kokinou, E., Papadimitriou, E., Karakostas, V., Kamberis, E. and Vallianatos, F., 2006. The Kefalonia Transform Zone (offshore Western Greece) with special emphasis to its prolongation towards the Ionian Abyssal Plain, Marine Geophysical Researches, 27(4), 241-252.

Malik, H.J., 1970. Estimation of the parameters of the Pareto distribution, Metrika, 15, 126-132.

Mann, H.B. and Whitney, D.R., 1947. On a Test of Whether one of Two Random Variables is Stochastically Larger than the Other, The Annals of Mathematical Statistics, 18(1), 50-60. 
Miller, J. and Adamchik, V.S., 1998. Derivatives of the Hurwitz Zeta function for rational arguments, Journal of Computational and Applied Mathematics, 100(2), 201-206.

Papadimitriou, E., 2002. Mode of Strong Earthquake Recurrence in the Central Ionian Islands (Greece): Possible Triggering due to Coulomb Stress Changes Generated by the Occurrence of Previous Strong Shocks, Bulletin of the Seismological Society of America, 92(8), 32933308.

Papazachos, B.C. and Comninakis, P.E., 1971. Geophysical and tectonic features of the Aegean Arc, Journal of Geophysical Research, 76(35), 8517-8533.

Patwardhan, A.S., Kulkarni, R.B. and Tocher, D., 1980. A semi-Markov model for characterizing recurrence of great earthquakes, Bulletin of the Seismological Society of America, 70(1), 323-347.

Sadeghian, R., Jalali-Naini, G.R., Sadjadi, J. and Fard, N.H., 2008. Applying Semi-Markov Models for forecasting the Triple Dimensions of Next Earthquake Occurrences: with Case Study in Iran Area, International Journal of Industrial Engineering \& Production Research, 19(4), 57-67.

Sadeghian, R., 2012. Forecasting time and place of earthquakes using a Semi-Markov model (with case study in Tehran province), Journal of Industrial Engineering International, 8(8), 1-7.

Scordilis, E.M., Karakaisis, G.F., Karacostas, B.G., Panagiotopoulos, D.G., Comninakis, P.E. and Papazachos, B.C., 1985. Evidence for Transform Faulting in the Ionian Sea: The Cephalonia Island Earthquake Sequence of 1983, Pure and Applied Geophysics, 123(3), 388-397.

Votsi, I., Limnios, N., Tsaklidis, G. and Papadimitriou, E., 2012. Estimation of the Expected Number of Earthquake Occurrences Based on Semi-Markov Models, Methodology and Computing in Applied Probability, 14(3), 685-703. 